\section{P127 CLINICAL PRESENTATION OF SYSTEMIC LUPUS ERYTHEMATOSUS IN PATIENTS WITH DIFFERENT GENOTYPES OF FOLATE CYCLE GENES}

N Panko*, N Shevchenko. Department of Paediatrics N 2, V.N. Karazin Kharkiv National University, Kharkiv, Ukraine

\subsection{6/annrheumdis-2018-EWRR2018.142}

Introduction Systemic Lupus Erythematosus (SLE) is a rheumatic multi-systemic chronic inflammatory disease in children, which is characterised by autoantibodies directed against selfantigens, immune complex formation, and immune dysregulation, resulting in damage to essentially any organ.

Objectives The aim was to assess the peculiarities of clinical presentation of systemic lupus erythematosus in children with different genotypes of folate cycle genes.

Methods The study involved 12 children with SLE aged from 7 to 18 years. There were 1 male and 11 female with disease duration from 7 month to 11 years. Patients were divided into 3 groups corresponding to the number of genes with risky genotypes. Group 1 included patients, who had one gene with risky genotype and 3 genes with neutral genotype; Group 2 consisted of children, who had 2 genes with risky genotype and 2 genes with neutral genotype; Group 3 included persons, who had 3 genes with risky genotype and 1 gene with neutral genotype. Assessment of children was based on physical, laboratory examination and instrumental tests. The measurement of genotypes of genes of folate cycle, such as 5-methyltetrahydrofolate-homocysteine methyltransferase (MTR), 5-methyltetrahydrofolate-homocysteine methyltransferase reductase (MTRR), 5,10-methylenetetrahydrofolate reductase C677T and A1298C variants (MTHFR-677 and MTHFR1298) were made by polymerase chain reaction. For the statistic processing of the material Stagraphics 3.0 for Windows was used.

Results Patients of Group 3 more frequently had risky genotype of MTHFR1298 gene (A/C or C/C) in comparison with other 2 groups $(\mathrm{p}<0.001)$, risky genotype of MTR $(\mathrm{A} / \mathrm{G}$ or $\mathrm{G} / \mathrm{G}$ ) and MTHFR677 (C/T or T/T) genes compared with Group $1(\mathrm{p}<0.001)$. Corresponding to analysis of anamnesis, disease onset in children of Group 1 the most common included in 33\% cases the skin syndrome (rash), arthritis and bleeding. $50 \%$ of patients of Group 2 had signs of nephritis as a prominent due to beginning of SLE that was more frequent than in Group $1(\mathrm{p}<0.001)$. Arthritis was determined as a main syndrome of disease onset in children of Group 3 $(66.7 \%)$, it was higher compared with Group $2(\mathrm{p}<0.001)$. At the moment of investigation general stage was assessed as a satisfactory the most frequent in patients of Group 3 than in others $(p<0.001)$. Nephritis and arthritis were the most common for children of Group 1 and $2(p<0.001)$. Neurologic symptoms and Antiphospholipid syndrome were more specific for patients of Group $2(50 \%, \mathrm{p}<0.001)$. Myocarditis was found on the average in $42 \%$ of children without statistical confidence between studying Groups.

Conclusions According to our findings, patients with different number of risky genotypes of folate cycle genes have different clinical presentation. The most severe syndromes were specific for children who had risky genotype of 2 folate cycle genes. The best case scenario of SLE was detected in patients who had risky genotype of 3 folate cycle genes.

Disclosure of interest None declared

\section{P128 ANALYSIS OF DNA METHYLATION PATTERNS IN RHEUMATOID ARTHRITIS PATIENT: A SYSTEM FOR PRIORITISING MEANINGFUL DIFFERENCE}

${ }^{1} \mathrm{R}$ Pitaksalee*, ${ }^{1}$ AN Burska, ${ }^{1} \mathrm{~J}$ Rogers, ${ }^{1} \mathrm{X}$ Xie, ${ }^{1} \mathrm{P}$ Emery, ${ }^{2} \mathrm{R}$ Hodgett, ${ }^{1} \mathrm{~F}$ Ponchel. ${ }^{1}$ Institute of Rheumatic and Musculoskeletal Medicine and NHR Leeds Musculoskeletal Biomedical Research Unit; ${ }^{2}$ Business Analysis and Decision Science, University of Leeds, Leeds, UK

10.1136/annrheumdis-2018-EWRR2018.143

Introduction Alterations in DNA methylation patterns have been related to several diseases, including Rheumatoid Arthritis (RA).

Objectives To identify changes in DNA methylation pattern of naïve and memory CD4 +T cells and monocytes in early, drug naïve RA patients to help understand early event in disease pathology.

Methods The methylation patterns of 480,000 CpGs were analysed in the 3 cell types from 6 healthy control (HC) and 10 RA patients using an Illumina genome-wide array. Standard t-tests were performed to associate p-value to individual CpGprobe. A scoring system was developed to select and prioritise differentially methylated CpGs with potential cumulative effect due to proximity with other significant CpGs. Rules for scoring were designed with respect to the significance of each CpGs and the distance (in bp) between them. Further filtering was applied to initially select the CpGs which highest significant ( $p$-value $\leq 0.0001)$ which have at least 2 proximal significant CpGs (p-value $\leq 0.01)$. Rules were coded in $R$ for systematic analysis. Lists of selected CpGs for all three cells for both hypo- and hyper-methylation were generated. Commonality was analysed using Venn diagram.

Results Different changes in methylation patterns were observed between HC and RA in the 3 cell types and thresholds of significance were set at p-value 0.01, 0.001 and 0.0001 . The total number of differentially methylated CpGs was enriched in naïve T-cells $(18,020)$ compared to memory $(14,197)$ and monocytes $(6,490)$ (p-value $\leq 0.01)$. Using our designed rules, we were able to prioritise cluster of differentially methylated CpGs which were then associated to 420, 7, and 48 hypomethylated genes, and 420, 719 and 21 hypermethylated genes respectively in naïve/memory T-cells and monocytes. Venn diagrams of hypermethylated gene showed only 1 genes (ABAT) in common to all 3 subsets. Hypomethylated gene showed no commonalities between the 3 cell subsets, and only 1 gene common between T-cell subsets (SLC43A2). Of note, the TNF gene was second on the priority list for naïve T-cell hypomethylation while no difference was observed in memory/monocytes. The IL-17 gene was de-methylated only in memory T-cells, hypermethylated in RA, but fully methylated in naïve/monocytes. Hypomethylation of socs3 was specific to monocytes.

Conclusions These data suggest quite different types of changes in patterns of DNA methylation affecting the 3 subsets early in the RA disease process. Our scoring system to prioritise clusters of differential methylation highlighted genes known to be related to early RA pathogenesis. Further work remains to explore the relationship between these genes and the biological effect at the transcriptional/translational level resulting from these alterations in DNA methylation.

Disclosure of interest None declared 